\title{
Screening of a Brassica napus bacterial artificial chromosome library using highly parallel single nucleotide polymorphism assays
}

\author{
Hieu Xuan Cao and Renate Schmidt ${ }^{*}$
}

\begin{abstract}
Background: Efficient screening of bacterial artificial chromosome (BAC) libraries with polymerase chain reaction (PCR)-based markers is feasible provided that a multidimensional pooling strategy is implemented. Single nucleotide polymorphisms (SNPs) can be screened in multiplexed format, therefore this marker type lends itself particularly well for medium- to high-throughput applications. Combining the power of multiplex-PCR assays with a multidimensional pooling system may prove to be especially challenging in a polyploid genome. In polyploid genomes two classes of SNPs need to be distinguished, polymorphisms between accessions (intragenomic SNPs) and those differentiating between homoeologous genomes (intergenomic SNPs). We have assessed whether the highly parallel Illumina GoldenGate ${ }^{\oplus}$ Genotyping Assay is suitable for the screening of a BAC library of the polyploid Brassica napus genome.

Results: A multidimensional screening platform was developed for a Brassica napus BAC library which is composed of almost 83,000 clones. Intragenomic and intergenomic SNPs were included in Illumina's GoldenGate ${ }^{\circledast}$ Genotyping Assay and both SNP classes were used successfully for screening of the multidimensional BAC pools of the Brassica napus library. An optimized scoring method is proposed which is especially valuable for SNP calling of intergenomic SNPs. Validation of the genotyping results by independent methods revealed a success of approximately $80 \%$ for the multiplex PCR-based screening regardless of whether intra- or intergenomic SNPs were evaluated.

Conclusions: Illumina's GoldenGate ${ }^{\circledast}$ Genotyping Assay can be efficiently used for screening of multidimensional Brassica napus BAC pools. SNP calling was specifically tailored for the evaluation of BAC pool screening data. The developed scoring method can be implemented independently of plant reference samples. It is demonstrated that intergenomic SNPs represent a powerful tool for BAC library screening of a polyploid genome.
\end{abstract}

Keywords: BAC library, Brassica napus, Genotyping, Multidimensional pools, PCR screening, Polyploidy, SNP

\section{Background}

Detailed genetic [1-3], physical mapping [4-7] and cytological studies $[8,9]$ provided evidence for a hexaploidization event that happened in the Brassica lineage after divergence from the Arabidopsis lineage. The extant diploid Brassica species, such as Brassica rapa, Brassica oleracea and Brassica nigra are therefore characterised by a complex genome structure. Brassica napus (AACC) is an amphidiploid species that was formed by hybridization of two Brassica species that diverged approximately 4 million years ago, Brassica rapa (AA) and Brassica oleracea

\footnotetext{
* Correspondence: schmidtr@ipk-gatersleben.de

Leibniz Institute of Plant Genetics and Crop Plant Research (IPK),

Corrensstraße 3, OT Gatersleben, D-06466 Stadt Seeland, Germany
}

(CC) $[10,11]$. The hybridization leading to Brassica napus is believed to be of recent origin. Most probably it dates back less than 10,000 years. Taking the evolutionary history of the Brassica species into account, every singlecopy gene in Arabidopsis thaliana should be present in three copies in the diploid Brassica species, in Brassica napus as much as six copies should be found. Microcollinearity studies revealed, however, that the triplicated genome segments that make up the diploid Brassica genomes have been subjected to many structural alterations, such as gene duplications, translocations, inversions, and especially frequently gene deletions. Due to these processes the gene numbers found in the Brassica species may show considerable deviations from the numbers stated above (summarized in [12]). 
A high-quality reference sequence for the Brassica napus genome is not yet available, thus studies of specific regions of the Brassica napus genome at sequence level have relied on the analysis of bacterial artificial chromosomes (BACs) or BAC contigs. Arabidopsis genes were used as probes to screen a Brassica napus BAC library by colony hybridization for genes and/or regions of interest, subsequently the BAC clones were assigned to different loci and characterised at sequence level $[13,14]$. These studies showed that the A and $C$ genomes in Brassica napus are very similar to those of the progenitors Brassica oleracea and Brassica rapa, however small differences with respect to the content of genes and mobile elements were observed. Thus, studies in Brassica napus can draw on resources that have been assembled for the progenitor genomes. For Brassica rapa a draft genome sequence was released [15] and for Brassica oleracea low coverage whole genome shotgun sequences were produced [16,17]. In addition, EST and genome survey sequences are available for different Brassica species (summarized in [18]).

The complex genome structure together with the high level of sequence identity of gene sequences in Brassica oleracea and Brassica rapa represent a considerable challenge for the identification of single nucleotide polymorphisms (SNPs) in Brassica napus [19]. Different classes of polymorphisms need to be considered. On the one hand SNPs will be found that occur between different accessions in either the $\mathrm{A}$ or the $\mathrm{C}$ genome. This class has been termed intragenomic SNP [20]. Intragenomic SNPs are readily suitable for genetic mapping and for the discrimination of accessions. Rarely SNP sites will be identified that are polymorphic in both genomes. On the other hand SNPs will be found that differentiate the homoeologous $\mathrm{A}$ and $\mathrm{C}$ genomes. This class has been referred to as interhomoeolog polymorphism [21] or intergenomic SNP [20]. Intergenomic SNPs are much more frequent than intragenomic SNPs [21], however they are neither useful for genetic mapping nor for the discrimination of accessions. Nonetheless, intergenomic SNPs may be particularly valuable for the screening of BAC libraries since they differentiate homoeologous sequences.

Initially amplicon sequencing of different accessions was used for SNP detection in Brassica napus [20,22,23]. Particularly large SNP collections were generated by transcriptome sequencing of different accessions [21] or by exploitation of sequence capture technology [24]. At first only few SNP markers were included in linkage maps [22,25], now high-density SNP maps are available for several Brassica napus mapping populations [24,26].

Illumina's GoldenGate ${ }^{\circledast}$ Genotyping Assay exploits oligonucleotide ligation and extension assays to distinguish allelic variants [27-29]. For each SNP locus three primers are designed that bind in the vicinity of the SNP. Two allele-specific oligonucleotides which carry the discriminating bases at their 3'-ends anneal upstream of the SNP while a common locus-specific primer matches sequences located downstream of the SNP. The locus-specific oligonucleotide carries in addition an Illumicode address sequence complementary to a particular bead type. In addition to the sequences which are specific for each locus analyzed the three different primers carry at their 5'-ends sequences which are specific for a particular oligonucleotide class. After hybridization of the oligonucleotides to the DNA templates to be analyzed a polymerase fills the gap between one of the allele- and the locus-specific oligonucleotide and a ligase seals the nick so that a contiguous template is formed. These reactions are carried out for many templates and loci simultaneously. Due to the presence of sequences specific for the three different oligonucleotide classes all templates can be amplified concurrently using just three primers. The specific polymerase chain reaction (PCR) primers that correspond to the two different allele-specific oligonucleotides are labelled with $\mathrm{Cy} 3$ and $\mathrm{Cy} 5$, respectively in order to allow allele differentiation. After amplification and downstream processing the single-stranded dye-labelled products are annealed to an optical array carrying beads that are coated with sequences complementary to the Illuminocode address sequences. The genotype at a given SNP locus is determined by analyzing the ratio of $\mathrm{Cy} 3$ and $\mathrm{Cy} 5$ fluorescence. For homozygous loci in diploid organisms near pure $\mathrm{Cy} 3$ or Cy5 fluorescence is observed, whereas both types of fluorescence are observed for heterozygous loci. In tetraploid organisms such as Brassica napus the situation is more complex. Assays which are specific for one of the genomes will produce $\mathrm{Cy} 3 / \mathrm{Cy} 5$ fluorescence ratios that are not distinguishable from those observed in diploid organisms. In contrast, assays which match target sequences on both homoeologous chromosomes may either segregate on one of the genomes or more rarely on both genomes. In these cases Cy3/Cy5 fluorescence ratios are generated that differ from those found in diploid organisms [20,30].

If BAC pools are analyzed for the presence of a particular intragenomic SNP locus with Illumina's GoldenGate ${ }^{\odot}$ Genotyping Assay it is expected that BAC pools that include one or more BACs corresponding to the SNP locus will show $\mathrm{Cy} 3$ or $\mathrm{Cy} 5$ fluorescence whereas $\mathrm{BAC}$ pools that consist solely of BACs that do not carry the locus should only give rise to residual fluorescence [31]. In case of intergenomic SNPs, BAC pools containing only one of the homoeologous loci should cause near pure $\mathrm{Cy} 3$ or $\mathrm{Cy} 5$ fluorescence whereas both types of fluorescence should be observed for those BAC pools harbouring both loci.

In this study it was tested whether Illumina's GoldenGate ${ }^{\oplus}$ Genotyping Assay can be used for the screening of a 
Brassica napus BAC library. In particular, it was evaluated whether both inter- and intragenomic SNPs are suitable for BAC library screening.

\section{Results and discussion}

\section{Isolation of Brassica napus gene sequences}

Seven Arabidopsis genes (At1g32440, At1g62640, At2g19450, At3g54320, At3g22960, At3g26790, At5g15530) were used as probes in colony hybridization experiments to identify all corresponding sequences in a Brassica napus BAC library of the genotype Express. All hybridizing clones were subjected to Southern blot analysis in order to confirm the results of the colony hybridizations and to assign the BAC clones to different loci. Between two and ten Brassica napus loci were found for the different candidate genes, in total 32 loci were identified. For one representative clone of each locus the Brassica napus sequences that corresponded to the genes of interest were determined ([32]; R. Schmidt, unpublished results). All Brassica napus gene sequences matching a particular Arabidopsis candidate gene were aligned and based on these alignments gene-specific oligonucleotide pairs were developed.

\section{Multidimensional screening platform of the Brassica napus BAC library}

The 82,944 clones of a Brassica napus BAC library were pooled according to the scheme shown in Figure 1.
Using this pooling system every clone of the library was represented once in each of six dimensions [33]. The 24 Brassica napus loci analyzed in this work were represented on average 10.6 times in the BAC library. Unambiguous deconvolution of clone coordinates using the six-dimensional pooling system depends on the depth of representation of a particular locus in the BAC library. In order to facilitate BAC coordinate deconvolution additional pooling dimensions were established. The SA and SB pools contain clones of six and eight 384-well plates, respectively (Figure 1). Moreover, pools containing clones of single plates with 384 BAC clones each were established. For library screening the six-dimensional pools were either analyzed together with the SA and SB pools (eight screening dimensions) or with the 216 single plate pools (seven screening dimensions).

In order to check the suitability of the established BAC pools for PCR-based screening, three gene-specific oligonucleotide pairs were used for PCR analyses with DNA samples of all six-dimensional BAC pools as well as the SA and SB pools as templates. Based on the results of the colony hybridization and locus assignment studies the pools which should harbour a particular gene-specific amplicon were deduced and served as reference for the results obtained by PCR. The predictions were found to be in good agreement with the experimental results (data not shown). Hence, both size and

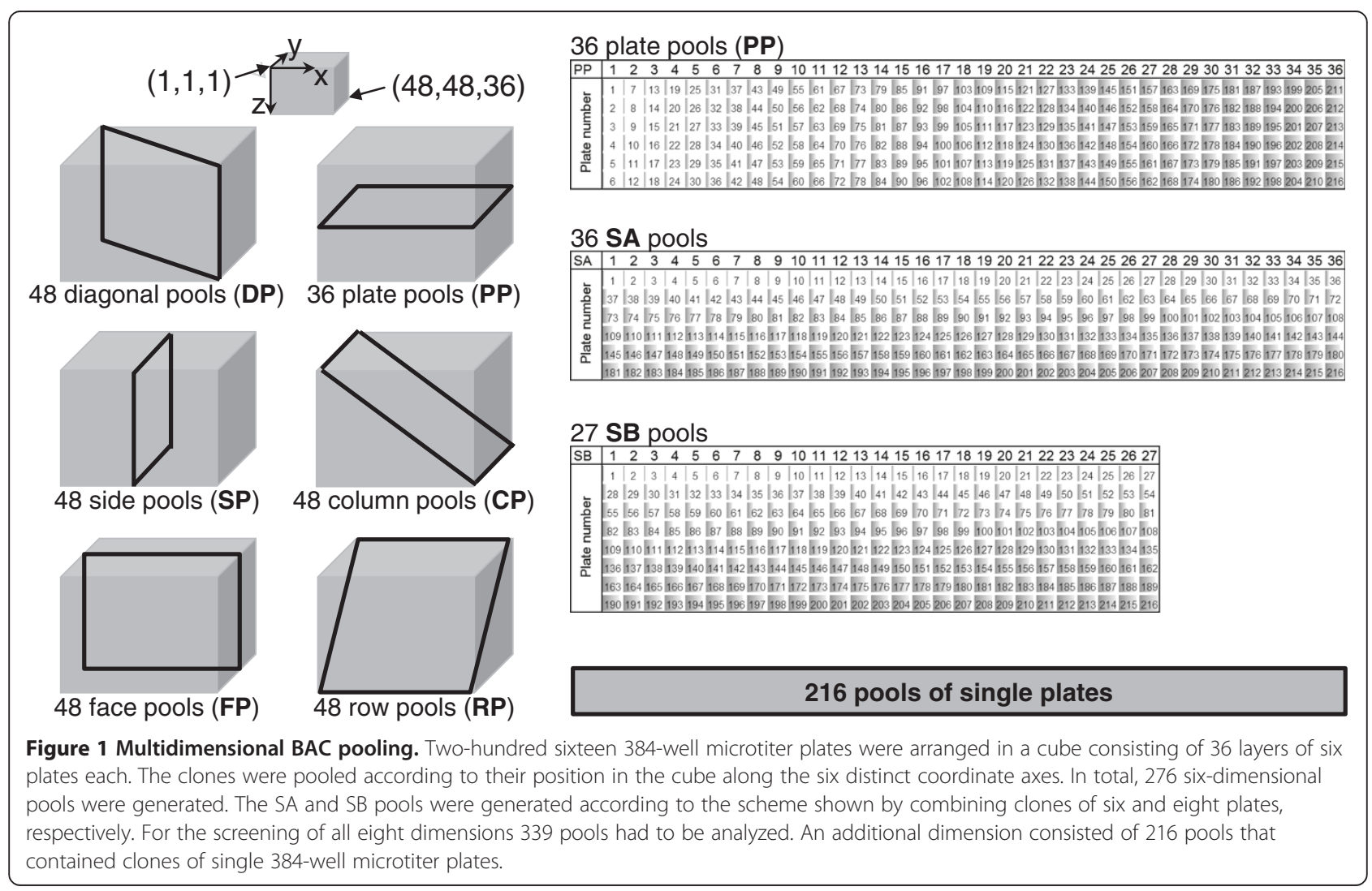


DNA quality of the different pools were suitable for PCR-based screening.

\section{Screening of the Brassica napus BAC library with Illumina's GoldenGate ${ }^{\oplus}$ genotyping assay}

In the majority of cases it was attempted to analyze the BAC pools for the presence/absence of individual Brassica napus genes. For each intragenomic SNP assay a single gene was assessed whereas intergenomic assays should allow the simultaneous detection and differentiation of homoeologous genes. The data set used in this study consisted of twenty intergenomic and fourteen intragenomic assays. In five additional cases the assays were designed in such a way that homoeologous genes would not be discriminated (Additional file 1, Additional file 2).

The 31 assays included in oligonucleotide pool assay 1 (OPA1) (Additional file 1, assays without the name affix "A") were used to screen all six-dimensional as well as the SA and SB BAC pools. DNA samples of Brassica accessions or pools of accessions were also analyzed.

SNP calling was performed with the Genotyping Module in GenomeStudio Data Analysis Software v2011.1. After normalization, signal intensities (normalized $\mathrm{R}$ ) and allele frequencies (normalized Theta) were plotted for all samples and assays. For each assay, matrices of signal thresholds were defined for the different genotypes using a clustering algorithm also referred to as genotype call areas. Based on this information the SNPs were called for the different samples. Examples are shown in Figure 2.

Luo et al. [31] reported that reliable scoring of BAC pools with oligonucleotide pool assays was possible provided that genotype call areas obtained for an Aegilops tauschii $\mathrm{F}_{2}$ population served as reference for the SNP calling of Aegilops tauschii BAC pools. This strategy was adapted for the data analysis in this study. In order to allow analysis of intra- and intergenomic SNPs DNAs of Brassica accessions rather than DNAs of a mapping population of Brassica napus were included as reference samples. In total, DNAs of forty different Brassica napus accessions, one Brassica napus $\mathrm{F}_{1}$-plant and four pools of Brassica accessions were analyzed. Genotype call areas were initially established based on the results of 34 Brassica DNA samples by the GenomeStudio Data Analysis Software v2011.1. These were then exported and used for the analysis of all samples. If appropriate, genotype call areas were manually adjusted.

The data for SNP assays \#52, \#51, \#47 that were developed for the analysis of two homoeologous genes, BnaA.PKp3.a and BnaC.PKp3.a, are shown as examples (Figure 2). The results of gene-specific assay \#52 (Figure 2A) were found to be in good agreement with the predictions based on the known coordinates that had been previously identified using colony hybridizations since all
71 pools which were known to contain BnaA.PKp3.a were determined (Figure 3). In addition, three pools which do not match any known BAC coordinate were also identified and classified as false positive scores. The genotyping results obtained for the pools of Brassica rapa and Brassica oleracea accessions were used to assign the different Brassica napus genes to the progenitor genomes (Additional file 1). Figure $2 B$ shows that the SNP of assay \#51 which is specific for gene BnaA.PKp3.a is present in the Brassica rapa accessions tested, whereas Brassica oleracea genotypes match the SNP specific for BnaC.PKp3.a. For gene-specific assay \#52 the Brassica oleracea accessions only give rise to residual fluorescence whereas the Brassica rapa accessions harbour the SNP specific for gene BnaA.PKp3.a.

The clustering algorithm did not perform equally well in case of intergenomic SNPs. The results for assays \#51 and \#47 exemplify this (Figure $2 \mathrm{~B}$ and $2 \mathrm{C}$ ). Assays \#47 and \#51 correctly determined $93 \%$ and $90 \%$, respectively of BAC pools that contained locus BnaA.PKp3.a, for locus BnaC.PKp 3.a lower detection rates of 82 and $85 \%$, respectively resulted (Figure 3 ). The eighteen BAC pools in which both homoeologous loci were present were in several cases not correctly assigned using the clustering algorithm, instead such pools were often ascribed to the call areas representing a single locus only (Figure $2 \mathrm{~B}$ and $2 \mathrm{C}$ ).

In contrast, the clustering algorithm was well-suited to identify plants that carried both loci of a particular intergenomic SNP. Brassica napus accessions were expected to contain both homoeologs, and indeed the clustering algorithm correctly assigned all Brassica napus accessions to the call area which corresponded to both SNPs in case of assay \#51 (Figure 2B). Also in case of intergenomic assay \#47 most Brassica napus accessions were found in the call area which indicated the presence of both SNPs in these plants (Figure 2C). However, some Brassica napus accessions were found to be homozygous for the SNP specific for BnaC.PKp3.a. These results were consistent with the finding that these Brassica napus accessions harboured an allele of BnaA.PKp3.a that was identical in sequence to BnaC.PKp3.a in the gene region corresponding to oligonucleotides 2 and 3 of assay \#47 (R. Schmidt, unpublished results). With this particular assay the pools of Brassica rapa and Brassica oleracea accessions were assigned to the same genotype. Moreover, the sequences of oligonucleotides 2 and 3 of assay \#47 perfectly match to the WGS sequences of Brassica rapa Chiifu-401 and Brassica oleracea O212 [34]. These results illustrate that intergenomic SNPs that were identified based on A and C genome sequences of few Brassica napus accessions are not necessarily capable to distinguish generally between $\mathrm{A}$ and $\mathrm{C}$ genomes.

The results for the plant samples presented here are consistent with other more comprehensive studies which 

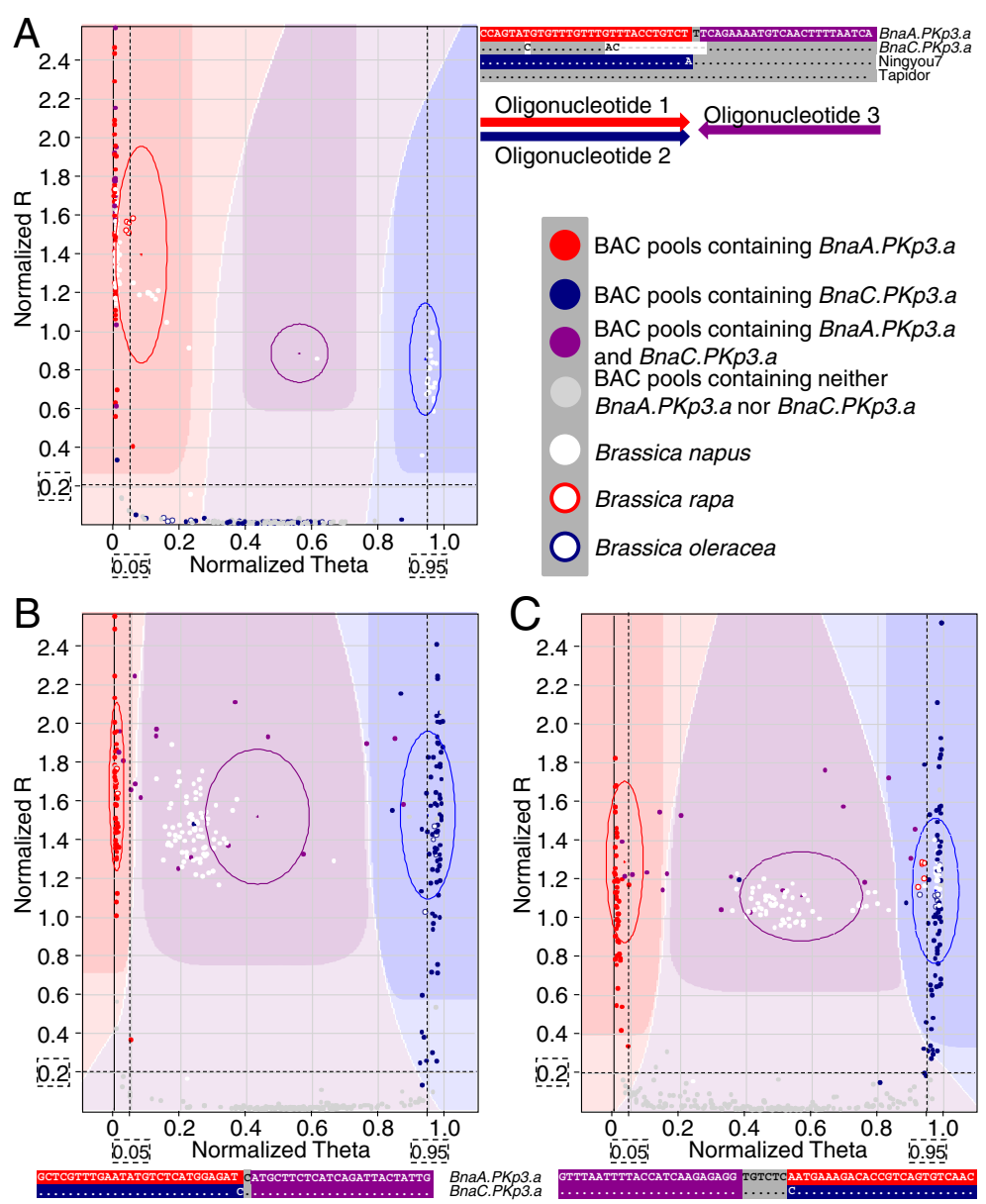

Figure 2 Analysis of BAC pools and Brassica accessions with oligonucleotide pool assays. The sequences represent those that were established for the genes found in the Express genome unless indicated otherwise. Panel (A) shows the results for SNP assay \#52 which was specific for BnaA.PKp3.a. In this intragenomic SNP assay oligonucleotide 1 corresponds to an allele which was found for example in the genotypes Express and Tapidor, whereas oligonucleotide 2 matched the allele present in the Ningyou7 genome. The darker colored regions in the graphs correspond to genotype call areas which were defined by GenomeStudio Data Analysis Software v2011.1, in the red and blue areas all plants homozygous for the Express and Ningyou7 alleles were found, respectively. Heterozygous plants showed up in the purple area. The results for SNP assays \#51 and \#47 are displayed in panels (B) and (C), respectively. Oligonucleotides 1 and 2 of intergenomic assays \#51 and \#47 discriminated between homoeologous genes BnaA.PKp3.a and BnaC.PKp3.a. In all graphs the data points for the BAC pools were color-coded according to the information which was deduced from the BAC coordinates that had been identified via colony hybridization and locus assignment studies. The dashed lines and boxed values correspond to the thresholds which were implemented for the optimized scoring method.

showed that genotype calling of plant samples by the GenomeStudio clustering algorithm is reliable and efficient (e.g. [20,31]), hence this methodology is widely used. In contrast, Luo et al. [31] concluded that the scoring of BAC pools was much more error-prone unless call areas established for a population of $\mathrm{F}_{2}$ plants provided a reference for the genotyping of BAC pools. In our study a considerable fraction of BAC pools were not assigned to the correct call areas with intergenomic SNPs, despite the fact that 45 Brassica DNA samples were included in the genotyping experiments. The data presented in Figure 2B and 2C show that an adjustment of genotype call areas which would correctly assign all
BAC pools to the different genotype classes is not possible because some of the normalized Theta values that belong to BAC pools that should give a signal with both SNPs are intermingled with those of BAC pools that harbour only one of the analyzed loci.

Loci and/or alleles are present in the vast majority of cases in certain ratios in a given plant accession whereas individual BAC clones will be present in unequal ratios in any given BAC pool. Furthermore, individual pools may not harbour equal numbers of BACs containing the homoeologous loci. These differences may account for the obvious discrepancy that has been detected with respect to scoring reliability of BAC pools and plant samples. 


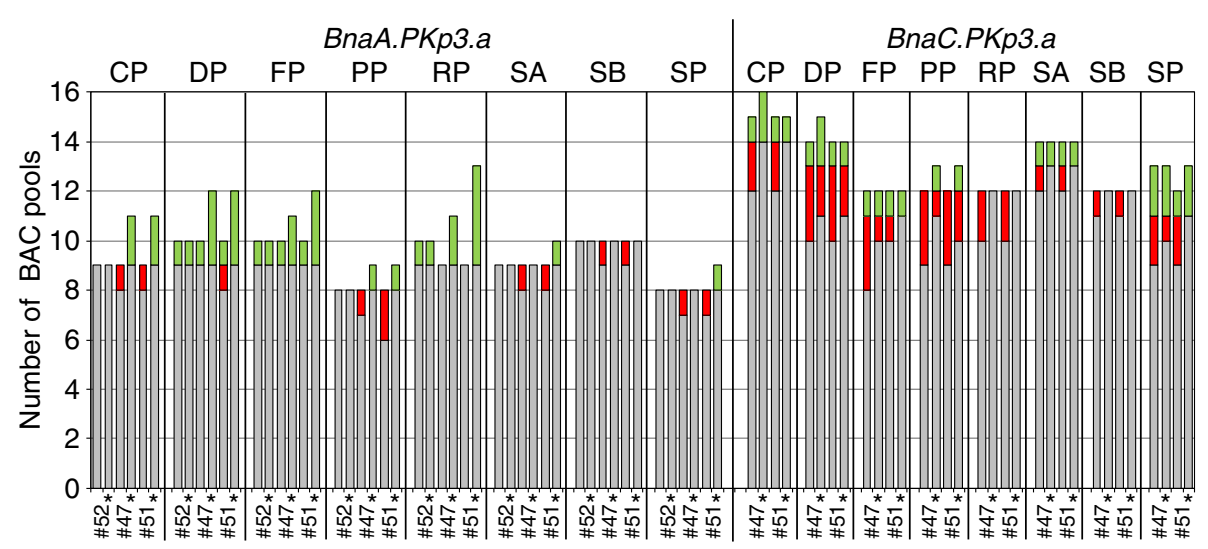

Figure 3 Scoring of BAC pools for the presence of SNPs with two methods. The coordinates of BAC clones which were shown to contain genes BnaA.PKp3.a and BnaC.PKp3.a according to the results of colony hybridizations and locus assignment studies served as reference for the scoring of the BAC pools with oligonucleotide pool SNP assays. The results of the optimized scoring method (for details please refer to the text) are marked with stars the other data were obtained with the clustering algorithm in GenomeStudio Data Analysis Software v2011.1. Grey shading indicates the number of BAC pools that were identified with a particular SNP assay (\#...) and corresponded to known BAC coordinates. Red boxes refer to the number of BAC pools representing known BAC coordinates but that were not detected with the SNP assays. Green shading highlights the number of identified pools that did not coincide with known BAC coordinates. The pools of the different dimensions are abbreviated in the same way as shown in Figure 1.

\section{Optimized SNP scoring method for BAC pools}

In order to improve the SNP genotyping of BAC pools an alternative scoring method was devised and evaluated. Only BAC pools which gave rise to normalized fluorescence intensity equal to or above 0.2 were classified as containing an SNP locus. Signals with normalized Theta values in the range from 0.05 to 0.95 were assigned to the category containing both SNPs, whereas values equal to or below 0.05 and equal to or above 0.95 were categorised to contain the alternative SNPs (Figure 2). These thresholds for the normalized Theta values were chosen based on the data that were obtained for 14 intragenomic assays (Additional file 2B) and for five assays that did not discriminate between homoeologous genes (Additional file 2C). The majority of BAC pools which showed fluorescence intensities equal to or above 0.2 had normalized Theta values between 0 and 0.05 or 0.95 and 1.0 , only $6 \%$ of the values were found in the range from 0.05 and 0.95 (Additional file 3A). An analysis of the individual assays revealed, however, differences. For seven assays none of the values were found in the interval between 0.05 and 0.95 , for another seven assays less than $2.5 \%$, but in two out of the 19 assays approximately $30 \%$ of values were observed in this interval (Additional file 3B).

Figures 2B, 2C and 3 show that several clone pools which were known to contain the loci of interest were identified with the optimized scoring system but not with the clustering algorithm. With the clustering algorithm 12 and 14\% of the expected BAC pools were not correctly assigned to the genotype call areas with assays \#51 and \#47, respectively. With the alternative scoring only $3 \%$ of the BAC pools were misclassified with each of the two assays.

The identification of a BAC coordinate requires a score in each of the screening dimensions, a scoring failure or a wrong SNP assignment in any of the dimensions will result in the complete loss of this particular BAC coordinate from the list of putative coordinates. Twenty-five BACs were known to carry loci BnaA.PKp.a or BnaC.PKp.a (Table 1). Using the clustering algorithm assays \#51 and \#47 identified only 11 (44\%) and 14 (56\%) BAC coordinates, respectively whereas the optimized scoring method revealed for assay \#51 22 (88\%) and for assay \#47 23 (92\%) coordinates. Thus, with the proposed alternative scoring method the frequency of false negative BAC coordinates was very much reduced. Moreover, this scoring scheme can be used independently of plant reference samples. Taking advantage of this, for the analysis of OPA2 a much smaller set of plant DNA samples was evaluated. In these experiments only the Brassica napus accession Express, the four different pools of Brassica accessions and two Brassica oleracea and Brassica rapa accessions each were examined.

The thresholds that were chosen to delineate the area which was categorised to carry both SNPs constituted a compromise. Figures 2 and 3 reveal that even the optimized scoring system did not identify all pools that harbour both loci. This could be fixed if the area which was classified to contain both SNPs would be increased. The consequences of an increased scoring area are seen in Figure 4. All 20 intergenomic SNPs that were analyzed in this study (Additional file 2A) were evaluated 
Table 1 Summary of SNP screening results for the Brassica napus BAC library

\begin{tabular}{|c|c|c|c|c|c|c|c|c|c|c|}
\hline Gene(s) & $\begin{array}{c}\text { Number } \\
\text { of known } \\
\text { BACs }\end{array}$ & $\begin{array}{l}\text { Number of } \\
\text { confirmed } \\
\text { BACs (\% of } \\
\text { known BACs) }\end{array}$ & $\begin{array}{c}\text { Number of } \\
\text { putative } \\
\text { BACs }\end{array}$ & $\begin{array}{c}\text { Number of } \\
\text { confirmed } \\
\text { BACs (\% of } \\
\text { putative BACs) }\end{array}$ & Assay & Gene(s) & $\begin{array}{c}\text { Number } \\
\text { of known } \\
\text { BACs }\end{array}$ & $\begin{array}{l}\text { Number of } \\
\text { confirmed } \\
\text { BACs (\% of } \\
\text { known BACs) }\end{array}$ & $\begin{array}{c}\text { Number of } \\
\text { putative } \\
\text { BACs }\end{array}$ & $\begin{array}{c}\text { Number of } \\
\text { confirmed } \\
\text { BACs (\% of } \\
\text { putative BACs) }\end{array}$ \\
\hline BnaA.PKp3.a & 10 & $10(100 \%)$ & 14 & $10(71.4 \%)$ & $\# 52$ & & & & & \\
\hline BnaA.PKp3.a & 10 & $10(100 \%)$ & 19 & $10(52.6 \%)$ & \#51 & BnaC.PKp3.a & 15 & $12(80 \%)$ & 38 & $12(31.6 \%)$ \\
\hline BnaA.PKp3.a & 10 & $10(100 \%)$ & 17 & $10(58.8 \%)$ & $\# 47$ & BnaC.PKpз.a & 15 & $13(86.7 \%)$ & 35 & 13 (37.1\%) \\
\hline BnaA.KAS III.a & 0 & 0 & 0 & 0 & $\# 7$ & BnaC.KAS III.a & 4 & $4(100 \%)$ & 5 & $4(80 \%)$ \\
\hline \multirow[t]{2}{*}{ BnaA.KAS III.a } & 0 & 0 & 0 & 0 & $\# 9$ & BnaC.KAS III.a & 4 & $4(100 \%)$ & 5 & $4(80 \%)$ \\
\hline & & & & & \#2 & BnaC.DGAT1.a & 15 & $13(86.7 \%)$ & 39 & $13(33.3 \%)$ \\
\hline BnaA.DGAT1.a & 2 & $1(50 \%)$ & 1 & $1(100 \%)$ & \#4 & BnaC.DGAT1.a & 16 & $14(87.5 \%)$ & 30 & $14(46.7 \%)$ \\
\hline BnaA.DGAT1.a & 2 & $1(50 \%)$ & 1 & 1 (100\%) & $\# 5$ & BnaC.DGAT1.a & 16 & 15 (93.8\%) & 33 & 15 (45.5\%) \\
\hline BnaA.DGAT1.b & 16 & 13 (81.3\%) & 33 & 13 (39.4\%) & \#9A & BnaC.DGAT1.b & 1 & 0 & 0 & 0 \\
\hline \multirow[t]{3}{*}{ BnaA.DGAT1.b } & 16 & 13 (81.3\%) & 38 & 13 (34.2\%) & $\# 10 \mathrm{~A}$ & BnaC.DGAT1.b & 1 & $1(100 \%)$ & 1 & $1(100 \%)$ \\
\hline & & & & & $\# 13 \mathrm{~A}$ & BnaC.DGAT1.c & 10 & $9(90 \%)$ & 10 & $9(90 \%)$ \\
\hline & & & & & $\# 14 \mathrm{~A}$ & BnaC.DGAT1.c & 10 & $7(70 \%)$ & 7 & 7 (100\%) \\
\hline BnaA.WRII.a & 5 & $4(80 \%)$ & 5 & $4(80 \%)$ & \#32 & BnaC.WR/1.a & 9 & 8 (88.9\%) & 11 & $8(72.7 \%)$ \\
\hline BnaA.WRI1.a & 5 & $4(80 \%)$ & 6 & $4(66.7 \%)$ & \#37 & BnaC.WRI1.a & 9 & 8 (88.9\%) & 11 & $8(72.7 \%)$ \\
\hline BnaA.WR/1.b & 14 & $13(92.9 \%)$ & 36 & $13(36.1)$ & \#28 & BnaC.WRI1.b & 11 & 11 (100\%) & 52 & $11(21.2 \%)$ \\
\hline BnaA.WRI1.b & 14 & 14 (100\%) & 534 & $14(2.6 \%)$ & $\# 30$ & BnaC.WRI1.b & 11 & 2 (18.2\%) & 13 & $2(15.4 \%)$ \\
\hline BnaA.PKp 1.a & 9 & 0 & 0 & 0 & $\# 7 \mathrm{~A}$ & & & & & \\
\hline BnaA.PKp 1.a & 9 & 1 (11.1\%) & 2 & $1(50 \%)$ & \#57 & BnaC.PKp 1.a & 7 & 7 (100\%) & 10 & $7(70 \%)$ \\
\hline BnaA.PKp 1.a & 9 & 0 & 0 & 0 & $\# 60$ & BnaC.PKp 1.a & 7 & $5(71.4 \%)$ & 16 & 5 (31.3\%) \\
\hline BnaA.PKp 1.a & 9 & $2(22.2 \%)$ & 2 & $2(100 \%)$ & $\# 3 \mathrm{~A}$ & BnaC.PKp 1.a & 7 & $4(57.1 \%)$ & 4 & $4(100 \%)$ \\
\hline \multirow[t]{4}{*}{ BnaA.PKp1.a } & 9 & $3(33.3 \%)$ & 3 & $3(100 \%)$ & $\# 8 \mathrm{~A}$ & BnaC.PKp 1.a & 7 & $2(28.6 \%)$ & 2 & $2(100 \%)$ \\
\hline & & & & & $\# 58$ & BnaC.PKp 1.a & 7 & $3(42.9 \%)$ & 3 & $3(100 \%)$ \\
\hline & & & & & \#55 & BnaC.PKp 1.b & 18 & $11(61.1 \%)$ & 45 & $11(24.4 \%)$ \\
\hline & & & & & $\# 56$ & BnaC.PKp 1.b & 18 & $13(72.2 \%)$ & 50 & $13(26 \%)$ \\
\hline BnaA.FUS3.a & 18 & $17(94.4 \%)$ & 108 & 17 (15.7\%) & $\# 14$ & BnaC.FUS3.a & 20 & $13(65 \%)$ & 77 & 13 (16.9\%) \\
\hline BnaA/C.FUS3.a & 38 & $36(94.7 \%)$ & 2492 & $36(1.4 \%)$ & $\# 10$ & & & & & \\
\hline BnaA/C.FUS3.a & 38 & 37 (97.4\%) & 3047 & 37 (1.2\%) & $\# 12$ & & & & & \\
\hline BnaA/C.FUS3.a & 38 & $36(94.7 \%)$ & 2249 & $36(1.6 \%)$ & $\# 18$ & & & & & \\
\hline BnaA/C.FUS3.a/b & 62 & 58 (93.5\%) & 12114 & $58(0.5 \%)$ & \#25 & & & & & \\
\hline BnaA.FUS3.b & 8 & $5(62.5 \%)$ & 10 & $5(50 \%)$ & $\# 20$ & BnaC.FUS3.b & 16 & $16(100 \%)$ & 38 & $16(42.1 \%)$ \\
\hline BnaA.FUS3.b & 8 & $8(100 \%)$ & 12 & $8(66.7 \%)$ & $\# 21$ & BnaC.FUS3.b & 16 & $14(87.5 \%)$ & 33 & $14(42.4 \%)$ \\
\hline BnaA.FUS3.b & 8 & $5(62.5 \%)$ & 9 & $5(55.6 \%)$ & \#22 & BnaC.FUS3.b & 16 & $16(100 \%)$ & 67 & 16 (23.9\%) \\
\hline BnaA.BCCP2.a & 12 & 11 (91.7\%) & 19 & 11 (57.9\%) & \#45 & & & & & \\
\hline \multirow[t]{3}{*}{ BnaA.BCCP2.a } & 12 & 10 (83.3\%) & 17 & $10(58.9 \%)$ & $\# 70$ & & & & & \\
\hline & & & & & \#43 & BnaC.BCCP2.a & 13 & $10(76.9 \%)$ & 36 & $10(27.8 \%)$ \\
\hline & & & & & $\# 44$ & BnaC.BCCP2.a & 13 & $11(84.6 \%)$ & 39 & $11(28.2 \%)$ \\
\hline BnaA.BCCP2.b & 9 & 9 (100\%) & 10 & $9(90 \%)$ & $\# 2 \mathrm{~A}$ & & & & & \\
\hline \multirow[t]{2}{*}{ BnaA С.BCCP2.b } & 21 & $20(95.2 \%)$ & 337 & $20(5.9 \%)$ & \#65 & & & & & \\
\hline & & & & & \#41 & BnaC.BCCP2.b & 12 & 11 (91.7\%) & 99 & 11 (11.1\%) \\
\hline
\end{tabular}



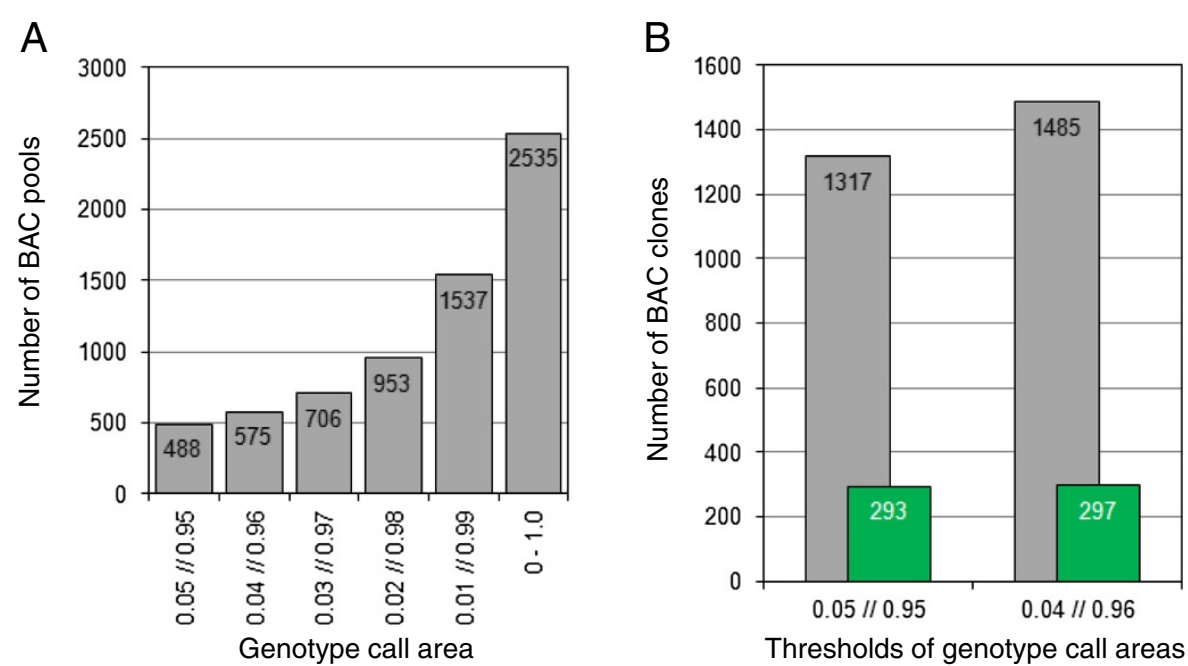

Figure 4 Defining the thresholds of the genotype call areas. The bars in panel (A) show how the thresholds for the genotype call areas affected the number of BAC pools that were assigned to the category containing both SNPs. In panel (B) the grey bars show the total number of putative BAC clones which resulted if two different sets of thresholds were applied to define the genotype call areas. The green bars correspond to the BAC clones which were confirmed to contain the loci of interest. Results for the 20 intergenomic assays listed in Additional file 2A were taken into account for the analysis represented in the two panels.

and it was calculated how many BAC pools would be classified as carrying both loci. Changing the scoring range from $0.05-0.95$ to $0.04-0.96$ resulted in 87 additional pools which were classified as containing both loci (Figure 4A). Due to this the number of putative BAC clones which needed to be assessed for the presence of the loci in additional experiments was increased by approximately $13 \%$. Thus, considerable more screening effort was needed whereas the number of clones that could be confirmed changed only marginally it increased by less than $1.5 \%$ (Figure 4B). Based on these results the thresholds for the genotype call areas were placed for all subsequent analyses at 0.05 and 0.95 .

\section{Assessment of screening results}

The putative BAC clones that were discovered with the oligonucleotide pool assays were analyzed with appropriate gene-specific amplicons in order to identify those clones that carried the loci of interest. A comparison of the coordinates of clones which were confirmed to contain the assayed loci with the list of BAC clones known to carry the loci of interest (Table 1, Additional file 2) revealed that multiplex PCR screening recovered $81.6 \%$ of the known BAC coordinates. The screening success of intergenomic SNPs was with $77.1 \%$ in the same range as that of genespecific SNPs (76.2\%). The five assays which did not discriminate between homoeologous genes in the Express genome even recovered $94.9 \%$ of the known clones.

For the experiments with OPA1 all six-dimensional pools as well as the SA and SB pools were screened, for the experiments with OPA2 all six-dimensional pools and the 216 single-plate pools were assessed. Thus, seven and eight screening dimensions were analyzed with OPA2 and OPA1, respectively. Taking into account that the identification of BAC coordinates relied on a score in each of the dimensions analyzed an overall success rate of more than $80 \%$ has to be regarded as very high. It can be concluded that a reliable screening platform has been established.

On average, only $5.8 \%$ of the pools which corresponded to known BAC coordinates failed to be detected because their normalized $\mathrm{R}$ values did not surpass the applied threshold. The summary of the screening results (Table 1) shows that not all loci were detected equally well. For example, the screening success for the BnaX.PKp1-loci, in particular for BnaA.PKp1.a, was lower than for the other genes of interest. For the assays corresponding to the BnaX.PKp1-loci the fraction of pools that escaped detection ranged from $9 \%$ for assay $\# 56$ to $75 \%$ for assay \#7A (Figure 5). As a consequence two of the assays (\#60, \#7A) designed for BnaA.PKP1.a did not reveal any of the nine $\mathrm{BAC}$ clones that contained this locus, three other assays (\#57, \#3A, \#8A) revealed between one and three of the known BAC coordinates (Table 1, Additional files $2 \mathrm{~A}$ and $2 \mathrm{~B})$. When it was attempted to recover the BAC clones containing BnaA.PKp1.a from the original glycerol stocks only few colonies could be retrieved whereas for most other clones many colonies were recovered when the glycerol stocks were spread on agar plates. These results suggest that clones that do not grow vigorously may be underrepresented in the pools and hence may escape detection.

In approximately half of the cases in which BAC clones were not identified, only one of the screening dimensions 


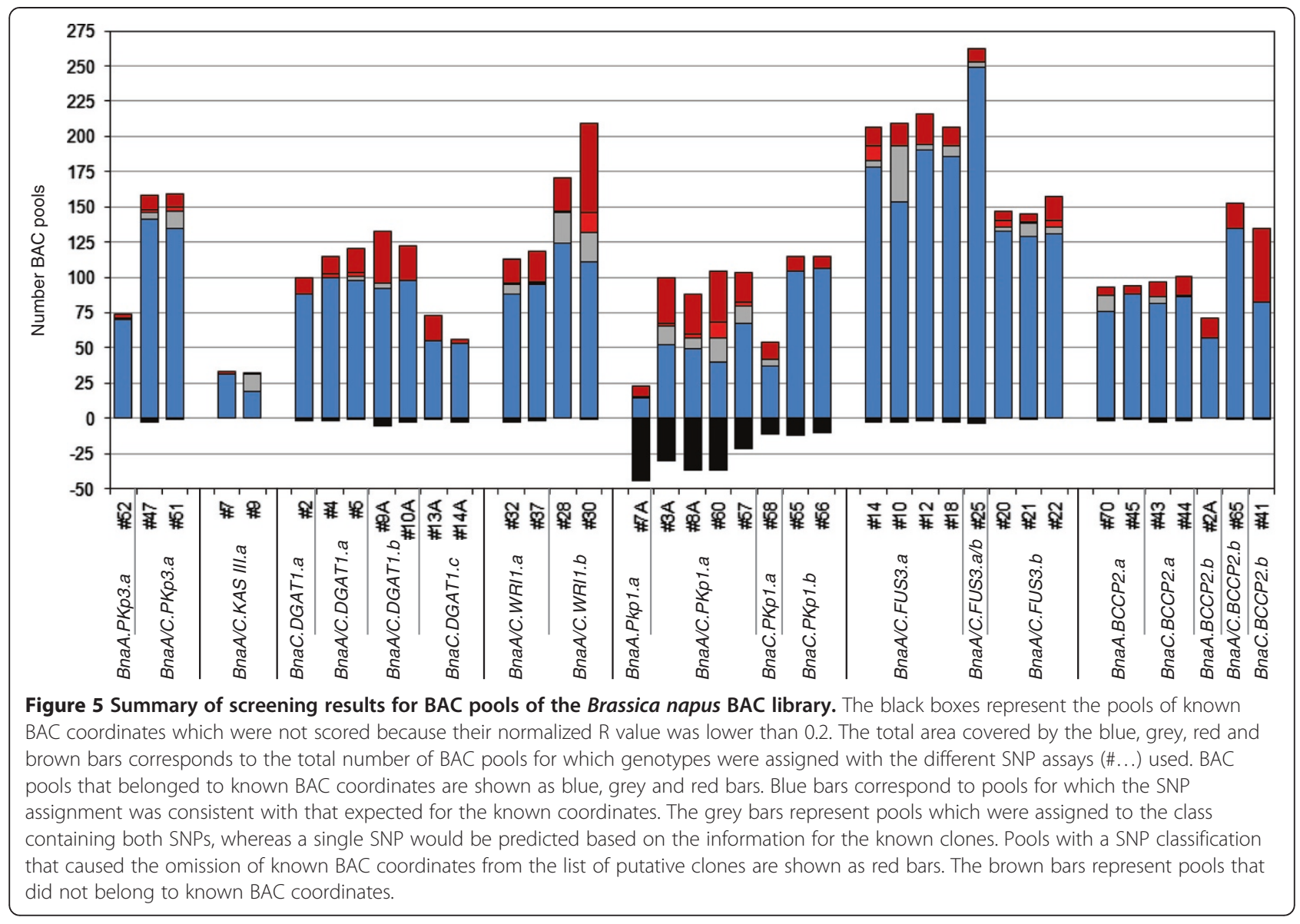

failed. In some instances false negative scores for a certain pool were observed with two or more independent assays corresponding to a particular gene whereas assays developed for other genes faithfully detected this pool. The BAC pools were established manually therefore it is possible that in rare cases individual clones were erroneously omitted from a pool and/or allocated to a wrong pool.

Assays \#2, \#4 and \#5 were designed to detect BnaC. DGAT1.a (Table 1, Additional files 1, 2A-B). For fifteen out of the sixteen clones known to contain this gene positive signals were found in at least seven out of the eight screening dimensions with all three assays. In contrast, clone 202N5 was only detected with assays \#4 and \#5, whereas assay \#2 did not reveal any signal specific for this clone. Analyses with gene-specific PCR amplicons confirmed that clone 202N5 carried sequences corresponding to assays \#4 and \#5. In contrast, sequences downstream of this region and where assay sequences \#2 were located were not present. This example shows the potential of multiplex-PCR screening for BAC contig building.

\section{Evaluation of the optimized SNP scoring method}

The reliability of the optimized scoring system was evaluated based on the entire data set (Figure 5). On average
$78 \%$ of all pools were assigned to the genotype that was expected for the known BAC coordinates. Another 7\% of the pools were assigned to both SNPs although the known BAC coordinates would predict only one of the SNPs. This type of SNP assignment did not lead to the omission of a known BAC coordinate from the list of putative clones, but in case of intergenomic SNPs it may lead to an increase in the number of putative clones that are identified. Cases in which the scoring method assigned a SNP that was in conflict with the expected genotype were rare, only $2 \%$ of the genotype scores were affected. Approximately $13 \%$ of the pools that were assigned to a genotype did not correspond to known BAC coordinates this type of false positive scores may increase the number of putative clones.

\section{Considerations for SNP assay design}

In this study all gene sequences corresponding to a particular Arabidopsis thaliana candidate gene were taken into consideration to select gene regions most suitable for SNP assays. The assays were developed such that the gene(s) and or homoeologs to be tested showed several mismatches and/or indels as close to the assay site as possible when compared to other homologs present in 
the Brassica napus genome. Consequently, overall an acceptable rate of false positive scores was observed, only few assays showed a high frequency of false positive scores. For assays \#41 and \#30 the proportion of false positive scores was particularly high, it accounted for approximately $40 \%$ of the detected pools (Figure 5).

With assay \#30 534 and 13 putative coordinates were detected for BnaA.WRI1.b and BnaC.WRI1.b, respectively. In contrast, assay \#28 revealed for the same loci 36 and 52 putative coordinates. With assay \#28 all 11 BAC clones known to be present for locus BnaC.WRI1.b in the library were detected, whereas assay \#30 revealed only 2 BAC clones for this locus (Table 1 ). Detailed analysis of the screening data for assay \#30 showed that 14 pools that should give a signal for BnaC.WRI1.b were only assigned to the category specific for BnaA.WRI1.b. These results suggest that the combination of oligonucleotides 1 and 3 of assay \#30 did not only detect BAC clones containing BnaA.WRI1.b but also additional loci that gave rise to scores that did not match known BAC coordinates of BnaA.WRI1.b and interfered with the correct assignment of pools containing BnaC.WRI1.b. Among the list of putative BAC clones which were revealed by assay \#30 for BnaA.WRI1.b several BAC coordinates were found that carried loci BnaA.WRI1.a and BnaC.WRI1.a. This implies that oligonucleotides 1 and 3 identified BAC pools containing BnaA.WRI1.a and BnaC.WRI1.a, although the assay sequences do not perfectly match to the sequences of these two loci. For oligonucleotide 3 one indel and one SNP were observed, both of which were more than $10 \mathrm{bp}$ upstream of the assay nucleotide. In case of oligonucleotide 1 an indel and a mismatch were found 6 and 9 bp downstream of the assay nucleotide. The detailed analysis of the results for assay \#30 stress that assay sequences should preferably contain multiple mismatches and/or indels very close to the assay site when compared to other homologs in order to avoid that homologous sequences interfere with the assay sequences in BAC library screens.

For many of the loci of interest the screen of the multidimensional screening platform resulted in a manageable list of putative BAC coordinates (Table 1). Thus, the use of seven or eight screening dimensions as described for OPA2 and OPA1 is suitable for a library with estimated 10-fold genome coverage. For loci for which ten or fewer BAC coordinates were known to be present in the library, in all but one case (\#60 for locus BnaC. PKp1.a) the fraction of clones that could be confirmed to contain the loci of interest made up 50\% or more of the putative coordinates. As expected, this fraction decreased if loci with more known coordinates were evaluated. The list of putative coordinates ranged from 13 to 108 when the loci were present between 11 and 20 times in the library, with the notable exception of data that were obtained with assay \#30 for locus BnaA.WRI1.b and that were already discussed. Assays that did not discriminate between homoeologs and that were aimed to detect between 21 and 62 coordinates revealed many hundred (\#65) or several thousand putative BAC coordinates (\#10, \#12, \#18,\#25). These results emphasize that it is essential to discriminate between homoeologous genes if a BAC library of a complexity similar to the one described here is used for PCR-based analyses.

\section{Conclusions}

Screening with multiplex SNP assays was successfully applied in the polyploid species Brassica napus for a BAC library with estimated 10-fold genome coverage. Intra- and intergenomic SNPs were equally suited for this purpose, provided that an optimized SNP calling method was implemented. Owing to the 10-fold coverage of the library only assays differentiating between (paleo)homoeologous genes proved to be suitable. For assays not discriminating between (paleo)homoeologous genes, even the use of one or two extra screening dimensions in addition to six-dimensional pools did not result in manageable lists of putative $\mathrm{BAC}$ coordinates. Our results suggest that BAC library screening with multiplex SNP assays can be effectively applied in polyploid species if sufficient sequence information is available for the identification of suitable SNPs.

\section{Methods}

Isolation and characterisation of Brassica napus gene sequences corresponding to selected Arabidopsis thaliana genes

Standard molecular biology techniques were performed as described in Sambrook et al. [35].

A Brassica napus BAC library of the genotype Express was constructed at Keygene N.V. (Wageningen, The Netherlands). The library consisted of almost 83,000 clones, arranged in 216 384-well microtiter plates. All clones of the library were gridded in duplicate and at high density on nylon membranes. Colony hybridizations using PCR-amplified Arabidopsis genes as probes were carried out according to O'Neill and Bancroft [4]. The DNA of all hybridizing clones was isolated, restricted with appropriate enzymes and subjected to Southern blot analyses with the Arabidopsis thaliana gene sequences as probes. Based on the resulting patterns the different BAC clones were assigned to different loci.

The analysis of 24 loci revealed an approximate 10fold genome coverage for the BAC library. For Brassica napus genome size estimates of 1130 to $1235 \mathrm{Mbp}$ were reported [36]. Consequently, the inserts of the BAC library should span on average between 135 and $150 \mathrm{kbp}$.

A representative BAC clone for each locus was selected for subcloning and sequencing of the Brassica 
napus genes that corresponded to a particular Arabidopsis thaliana candidate gene. Sequencing was performed by primer walking and carried out at Eurofins MWG Operon (Ebersberg, Germany) or at the IPK Gatersleben (Germany). Oligonucleotides were purchased from Eurofins MWG Operon (Ebersberg, Germany). Nomenclature of the genes followed the standard proposed by Østergaard and King [37].

\section{Development of gene-specific amplicons}

All Brassica napus gene sequences that corresponded to a particular Arabidopsis thaliana candidate gene were aligned using the CHAOS + Dialign webserver [38]. These alignments provided the basis for the design of gene-specific oligonucleotide pairs. Primers with an optimum melting temperature of $60^{\circ} \mathrm{C}$ were selected with Primer3 (Whitehead Institute for Biomedical Research, Cambridge MA; [39]).

DNA samples of BAC clones that represented all different Brassica napus loci corresponding to a particular candidate gene were used as templates in PCR experiments in order to identify the annealing temperature at which gene-specific products were obtained for each oligonucleotide pair. These optimized temperature profiles were then used for PCR amplification with total DNA of the cultivar Express as template. Only if direct sequencing of the resulting PCR product confirmed that a particular primer pair amplified a fragment of a single gene it was included in the genetic diversity studies.

\section{SNP discovery}

DNA samples derived from a set of Brassica napus accessions served as templates for PCR amplifications with the gene-specific oligonucleotide pairs and the PCR products were directly sequenced. The resulting sequences were manually edited and subsequently aligned with BioEdit [40]. The sequence alignments were inspected for the presence of SNPs. The genetic diversity studies were performed for homoeologous Brassica napus genes, it was therefore possible to identify and distinguish intragenomic and intergenomic SNPs. The results of the genetic diversity studies will be reported elsewhere.

\section{Multidimensional pooling strategy}

A six-dimensional pooling strategy as described by Klein et al. [33] was adopted for the Brassica napus BAC library of the genotype Express. The 216 384-well microtiter plates that make up the BAC library were conceptually arranged in a cube consisting of 36 layers, 48 columns, and 48 rows, thus each layer consisted of six 384-well plates. The clones were then pooled according to their position in the cube along the six distinct coordinate axes to generate 36 plate pools (PP), 48 diagonal pools (DP), 48 side pools (SP), 48 column pools (CP), 48 face pools (FP) and
48 row pools (RP). The plate pools consisted of 2304 clones each, whereas 1728 clones each made up the pools of the other five dimensions (Figure 1).

Plate pools were generated by transferring clones from the six 384-well plates which make up one layer of the cube with a 384-pin tool into a single plate. To generate the pools of the other dimensions in the most efficient manner first suitable master pools were assembled which contained all clones of up to six particular microtiter plates. For the construction of the master plates for the side pools, face pools and diagonal pools, for example, all plates which were present in the same position in six consecutive layers of the cube were combined. Depending on which of the dimensions was prepared 24-, 16-well pin tools or toothpicks were used to transfer the appropriate BAC clones from the master pool plates into the stock plate for each particular pool.

Stocks for each individual pool were generated in a single 384-well microtiter plate containing $70 \mu \mathrm{l}$ of glycerol-containing bacterial growth medium plus 12.5 $\mu \mathrm{g} / \mathrm{ml}$ chloramphenicol per well. After incubation for $16-20 \mathrm{~h}$ at $37^{\circ} \mathrm{C}$ all cultures of a single 384 -well microtiter plate were combined in sterile containers and aliquots were stored at $-80^{\circ} \mathrm{C}$ until further use.

For the SA and SB pools clones of six (36 pools à 2304 BAC clones) and eight entire microtitre plates each (27 pools à 3072 BAC clones) were combined according to the scheme shown in Figure 1. In addition, 216 pools containing clones of single plates with 384 BAC clones each were established.

\section{BAC pool DNA isolation}

The glycerol stocks of the individual pools were used to inoculate $100 \mathrm{ml}$ of liquid bacterial growth medium supplemented with $12.5 \mu \mathrm{g} / \mathrm{ml}$ chloramphenicol. Growth took place for $16-20 \mathrm{~h}$ in a gyratory shaker at $37^{\circ} \mathrm{C}$. BAC DNA isolation was performed with NucleoBond ${ }^{\circ}$ PC100 (Macherey-Nagel GmbH \& Co. KG, Düren, Germany) according to manufacturer's instructions for the six-dimensional pools as well as the SA and SB pools. For the 216 DNA pools that were generated from single plates $5 \mathrm{ml}$ of bacterial culture each were used for DNA extractions with the GeneJET ${ }^{\mathrm{rm}}$ Plasmid Miniprep Kit (Fermentas, St. Leon Rot, Germany). Quantification of the DNA samples was performed with the Quant-iT ${ }^{\mathrm{m}}$ PicoGreen ${ }^{\bullet}$ dsDNA Assay Kit (Life Technologies GmbH, Darmstadt, Germany).

\section{PCR conditions}

PCR was carried out in reaction volumes of $30 \mu \mathrm{l}$ containing approximately $5 \mathrm{ng}$ BAC-pool DNA, $1 \times$ DreamTaq $^{\text {Tm }}$ buffer, $250 \mu \mathrm{M}$ dNTP, 30 pmol of each primer and $1 \mathrm{U}$ of DreamTaq ${ }^{\circledR}$ DNA Polymerase (Fermentas, St. Leon Rot, Germany). All samples were preheated for 
$10 \mathrm{~min}$ at $95^{\circ} \mathrm{C}$ and then subjected to 35 PCR cycles. The cycles consisted of a denaturation step at $94^{\circ} \mathrm{C}$ for $30 \mathrm{~s}$, an annealing step for $30 \mathrm{~s}$, and an elongation step at $72^{\circ} \mathrm{C}$ for $1 \mathrm{~min}$. After a final extension of $5 \mathrm{~min}$ at $72^{\circ} \mathrm{C}$ the reactions were cooled down to $15^{\circ} \mathrm{C}$. Aliquots of $5 \mu$ l were resolved on agarose gels in $1 \times$ TBE.

In order to assay single BAC clones for the presence or absence of a particular amplicon single bacterial colonies were transferred to PCR tubes containing all necessary reagents. Conditions for PCR were the same as described above.

In case Brassica napus DNA samples were used for PCR the reactions were carried out in $50 \mu \mathrm{l}$ and approximately $20 \mathrm{ng}$ of template DNA were used for amplification. Prior to sequencing the PCR products were purified with appropriate spin columns (peqGOLD MicroSpin Cycle-Pure Kit, PEQLAB Biotechnologic $\mathrm{GmbH}$, Erlangen, Germany).

\section{Analysis with Illumina's GoldenGate ${ }^{\circledast}$ genotyping assay}

Sequences containing suitable SNPs were extracted and supplied to Illumina Inc. (San Diego, CA) for design of multiplex oligonucleotide pool assays (OPA, Additional file 1). The 31 assays without the name affix " $A$ " were part of OPA1, the remaining eight assays were part of OPA2. All assays used had a designability score higher than 0.6 .

All experimental steps were carried out as recommended by the manufacturer and the fluorescence signals were recorded with a BeadXpress Reader (Illumina Inc. San Diego, CA).

Initially three different DNA template amounts were tested for 20 BAC pools each in a multiplex GoldenGate ${ }^{\circledast}$ Genotyping Assay. Similar results were obtained regardless whether 50,125 or 250 ng were used as DNA input. In all subsequent experiments $125 \mathrm{ng}$ template DNA were used for each pool that contained between 1728 and 3072 clones. For all BAC pools that harboured DNA of 384 clones each lower amounts of template DNA were used. Tests with six such BAC pools showed that comparable results were obtained with 5, 10 and $25 \mathrm{ng}$ of template DNA. In all subsequent experiments $10 \mathrm{ng}$ of template DNA served as DNA input for the BAC pools of single 384-well plates.

Plant DNA samples were amplified with the Illustra Genomi Phi V2 DNA Amplification Kit (GE Healthcare Europe $\mathrm{GmbH}$, Munich, Germany) and $250 \mathrm{ng}$ each of the resulting reaction products were taken as templates for genotyping.

For the experiments with OPA1 all six-dimensional pools as well as the SA and SB pools were analyzed alongside with DNA of 40 Brassica napus accessions, a Brassica napus $\mathrm{F}_{1}$-plant and four pools of Brassica accessions. Two of the pools consisted of summer and winter oilseed rape lines, the other two pools were comprised of Brassica oleracea and Brassica rapa accessions. Initially, the GenomeStudio Data Analysis Software v2011.1 (Illumina Inc., San Diego, CA) was used for calling of genotypes with default parameters. The genotype call areas were initially defined based on the analysis of 29 DNAs of Brassica napus accessions, one $\mathrm{F}_{1}$-plant and the four different DNA pools of Brassica accessions. The clusters for the three different genotypes were exported from the plots to serve as reference for the analysis of the BAC pool DNA samples. Based on the data of all DNA samples the genotype call areas were manually adjusted, if needed.

Subsequently, an optimized scoring system for BAC pools was developed. Only BAC pools which showed normalized fluorescence intensities (Normalized R) equal to or higher than 0.2 were classified as carrying a particular SNP. BAC pools showing signals with normalized Theta values (Normalized Theta) equal to or below 0.05 and equal to or above 0.95 were classified to contain the alternative SNPs, whereas values in the range from 0.05 to 0.95 were assigned to the category containing both SNPs.

For the experiments with OPA2 all six-dimensional pools and the 216 pools of single 384-well plates were examined alongside the DNA of Brassica napus accession Express, two Brassica oleracea accessions, two Brassica rapa accessions and the DNA of the four pools of Brassica accessions.

\section{Clone deconvolution}

BAC coordinate deconvolution was established in Microsoft Excel (Microsoft Corporation, Redmond, USA). For all 82,944 BAC coordinates the corresponding pool information was entered into an Excel table. All pools that contained a particular SNP were selected with the filter function in order to deduce the list of all putative BAC coordinates for this assay nucleotide.

\section{Analysis of putative BAC coordinates}

The BAC clones which were identified for the different assays were analyzed with the help of gene-specific amplicons (Additional file 4) for the presence of the different Brassica napus loci. The validation of the results which resulted from the screening of all six-dimensional pools as well as the SA and SB pools (OPA1) was carried out in two steps. Based on the list of putative BAC coordinates all 384-well plates which contained these clones were selected and DNAs of these pools were used as templates in PCR assays with gene-specific primer pairs in order to identify those plates which contained a particular locus. Only putative BAC clones which were present in these plates were tested individually for the presence of a particular locus by PCR. In cases in which 
several hundreds or thousands of putative clones were identified it was only analyzed whether the known coordinates for the corresponding loci were among the list of putative clones.

For the assays of OPA2 all putative BAC coordinates which resulted from the screening of the sixdimensional pools and the 216 pools of the individual microtiter plates were screened by PCR with genespecific amplicons (Additional file 4) for the presence of a particular locus.

All clones that were confirmed to contain loci of interest were included in the list of BAC coordinates known to be present in the library.

\section{Additional files}

Additional file 1: Sequence information for oligonucleotide pool

assays. Bold font indicates sequences specific for a particular

oligonucleotide class. Illuminocode sequences are shown in italics. Plain font corresponds to sequences of Brassica napus alleles and/or the specified genes. Assays with the name affix " $\mathrm{A}$ " were included in experiments with oligonucleotide pool assays (OPA) 2 the remainder were part of OPA1.

Additional file 2: Clones detected in the BAC library. (A) Screening results for SNP assays that discriminated between homoeologous genes in the Express genome. (B) Screening results for SNP assays specific for single genes in the Express genome. (C) Screening results for SNP assays that did not differentiate between homoeologous genes in the Express genome.

Additional file 3: Distribution of normalized Theta values. Panel (A) shows which proportion of pools that revealed normalized $R$ values of 0.2 or higher were found in the indicated intervals of normalized Theta values. In order to integrate the data of all assays the normalized Theta values were transformed as follows: $0.5-$ |0.5-normalized Theta|. Grey bars represent data for assays that were specific for single genes in the Express genome (Additional file 2B) and white bars correspond to data for those SNP assays that did not differentiate between homoeologous genes in the Express genome (Additional file 2C). The grey and black bars in panel (B) indicate for each of the assays analyzed which proportion of BAC pools was assigned to the class containing a single SNP (normalized Theta $\leq 0.05$ or normalized Theta $\geq 0.95$ ) or both SNPs $(0.05<$ normalized Theta $<0.95)$, respectively.

Additional file 4: Gene-specific amplicons. The table lists all amplicons which were used to analyze the putative BAC clones for the presence of a particular locus by PCR. The oligonucleotides belonging to amplicon BnaA/C.DGAT1.b amplified in Brassica napus Express BnaA. DGAT1.b and BnaC.DGAT1.b.

\section{Competing interests}

The authors declare that they have no competing interests.

\section{Authors' contributions}

HXC and RS planned the work. HXC oversaw and participated in the construction of the BAC pools, carried out and evaluated the genotyping, deconvoluted the BAC coordinates, validated the results by PCR and prepared figures. RS conceived the study, contributed to identification and characterization of Brassica napus gene sequences, designed gene-specific amplicons, selected the SNP information for the genotyping assays and drafted the manuscript. Both authors read and approved the final draft of the manuscript.

\section{Acknowledgments}

This work was supported by BMBF grant KMU-innovativ 4 (0315639B). Isolation of the Brassica napus gene sequences and the genetic diversity studies were funded through BMBF grants GABI-BRIDGE (0312860B) and GABI-OIL (0315053G). The authors thank Katrin Bach for providing BnaX. DGAT1, BnaX.WRI1 and BnaX.BCCP2 gene sequences. Kristin Langanke and Eva Arlt are acknowledged for their skillful assistance in the isolation of the Brassica napus gene sequences and the genetic diversity studies and Kathleen Herz for help with the preparation of BAC pools and PCR analyses. The authors express their gratitude to Martin Ganal, Gregor Durstewitz and Sandra Reis (TraitGenetics GmbH, Germany) for helpful advice on assay design and the Veracode platform and for providing some of the plant DNA samples which served as references in the Illumina assays. Frank Breuer and Milena Ouzunova (KWS Saat AG, Germany) provided seed material of Brassica napus accessions. The authors thank Giang Vu for comments on the manuscript.

Received: 14 May 2013 Accepted: 23 August 2013

Published: 6 September 2013

\section{References}

1. Lagercrantz U, Lydiate DJ: Comparative genome mapping in Brassica. Genetics 1996, 144:1903-1910.

2. Lagercrantz U: Comparative mapping between Arabidopsis thaliana and Brassica nigra indicates that Brassica genomes have evolved through extensive genome replication accompanied by chromosome fusions and frequent rearrangements. Genetics 1998, 150:1217-1228.

3. Parkin IA, Gulden SM, Sharpe AG, Lukens L, Trick M, Osborn TC, Lydiate DJ: Segmental structure of the Brassica napus genome based on comparative analysis with Arabidopsis thaliana. Genetics 2005, 171:765-781.

4. O'Neill CM, Bancroft I: Comparative physical mapping of segments of the genome of Brassica oleracea var. alboglabra that are homoeologous to sequenced regions of chromosomes 4 and 5 of Arabidopsis thaliana. Plant J 2000, 23:233-243.

5. Rana D, van den-Boogaart T, O'Neill CM, Hynes L, Bent E, Macpherson $L$, Park JY, Lim YP, Bancroft I: Conservation of the microstructure of genome segments in Brassica napus and its diploid relatives. Plant J 2004, 40:725-733.

6. Park JY, Koo DH, Hong CP, Lee SJ, Jeon JW, Lee SH, Yun PY, Park BS, Kim HR, Bang JW, Plaha P, Bancroft I, Lim YP: Physical mapping and microsynteny of Brassica rapa ssp. pekinensis genome corresponding to a $222 \mathrm{kbp}$ gene-rich region of Arabidopsis chromosome 4 and partially duplicated on chromosome 5. Mol Genet Genomics 2005, 274:579-588.

7. Navabi ZK, Huebert T, Sharpe AG, Bancroft I, Parkin IA, Neill CM O: Conserved microstructure of the Brassica B Genome of Brassica nigra in relation to homologous regions of Arabidopsis thaliana, B. rapa and B. oleracea. BMC Genomics 2013, 14:250

8. Lysak MA, Koch MA, Pecinka A, Schubert I: Chromosome triplication found across the tribe Brassiceae. Genome Res 2005, 15:516-525.

9. Lysak MA, Cheung K, Kitschke M, Bures P: Ancestral chromosomal blocks are triplicated in Brassiceae species with varying chromosome number and genome size. Plant Physiol 2007, 145:402-410.

10. $U$ N: Genomic analysis in Brassica with special reference to the experimental formation of $B$. napus and peculiar mode of fertilisation. Jpn J Bot 1935, 7:389-452.

11. Inaba R, Nishio T: Phylogenetic analysis of Brassiceae based on the nucleotide sequences of the S-locus related gene, SLR1. Theor Appl Genet 2002, 105:1159-1165.

12. Town C, Schmidt R, Bancroft I: Comparative genome analysis at the sequence level in the Brassicaceae. In Genetics and Genomics of the Brassicaceae. Edited by Schmidt R, Bancroft I. New York Dordrecht Heidelberg London: Springer; 2011:171-194.

13. Cheung F, Trick M, Drou N, Lim YP, Park JY, Kwon SJ, Kim JA, Scott R, Pires JC, Paterson AH, Town C, Bancroft I: Comparative analysis between homoeologous genome segments of Brassica napus and its progenitor species reveals extensive sequence-level divergence. Plant Cell 2009, 21:1912-1928.

14. Cho K, O'Neill CM, Kwon SJ, Yang TJ, Smooker AM, Fraser F, Bancroft !: Sequence level comparative analysis of the Brassica napus genome around two stearoyl-ACP desaturase loci. Plant J 2010, 61:591-599.

15. The Brassica rapa Genome Sequencing Project Consortium: The genome of the mesopolyploid crop species Brassica rapa. Nat Genet 2011, 43:1035-1039 
16. Ayele M, Haas BJ, Kumar N, Wu H, Xiao Y, Van Aken S, Utterback TR, Wortman JR, White OR, Town CD: Whole genome shotgun sequencing of Brassica oleracea and its application to gene discovery and annotation in Arabidopsis. Genome Res 2005, 15:487-495.

17. Katari MS, Balija V, Wilson RK, Martienssen RA, McCombie WR: Comparing low coverage random shotgun sequence data from Brassica oleracea and Oryza sativa genome sequence for their ability to add to the annotation of Arabidopsis thaliana. Genome Res 2005, 15:496-504.

18. Schmidt R, Bancroft I: Perspectives on Genetics and Genomics of the Brassicaceae. In Genetics and Genomics of the Brassicaceae. Edited by Schmidt R, Bancroft I. New York Dordrecht Heidelberg London: Springer; 2011:617-632

19. Kaur S, Francki MG, Forster JW: Identification, characterisation and interpretation of single-nucleotide sequence variation in allopolyploid crop species. Plant Biotech J 2012, 10:125-138.

20. Durstewitz G, Polley A, Plieske J, Luerssen H, Graner EM, Wieseke R, Ganal MW: SNP discovery by amplicon sequencing and multiplex SNP genotyping in the allopolyploid species Brassica napus. Genome 2010, 53:948-995.

21. Trick M, Long Y, Meng J, Bancroft I: Single nucleotide polymorphism (SNP) discovery in the polyploidy Brassica napus using Solexa transcriptome sequencing. Plant Biotech J 2009, 7:334-346.

22. Qiu D, Morgan C, Shi J, Long Y, Liu J, Li R, Zhuang X, Wang Y, Tan X, Dietrich E, Weihmann T, Everett C, Vanstraelen S, Beckett P, Fraser F, Trick M, Barnes S, Wilmer J, Schmidt R, Li J, Li D, Meng J, Bancroft I: A comparative linkage map of oilseed rape and its use for QTL analysis of seed oil and erucic acid content. Theor Appl Genet 2006, 114:67-80.

23. Westermeier P, Wenzel G, Mohler V: Development and evaluation of single-nucleotide polymorphism markers in allotetraploid rapeseed (Brassica napus L.). Theor Appl Genet 2009, 119:1301-1311.

24. Delourme R, Falentin C, Fopa Fomeju B, Boillot M, Lassalle G, André I, Duarte J, Gauthier V, Lucante N, Marty A, Pauchon M, Pichon J-P, Ribière N Trotoux G, Blanchard P, Rivière N, Martinant J-P, Pauquet J: High-density SNP based genetic map development and linkage disequilibrium assessment in Brassica napus L. BMC Genomics 2013, 14:120.

25. Hu Z, Huang S, Sun M, Wang H, Hua W: Development and application of single nucleotide polymorphism markers in the polyploid Brassica napus by 454 sequencing of expressed sequence tags. Plant Breeding 2012 131:293-299.

26. Bancroft I, Morgan C, Fraser F, Higgins J, Wells R, Clissold L, Baker D, Long Y, Meng J, Wang X, Liu S, Trick M: Dissecting the genome of the polyploid crop oilseed rape by transcriptome sequencing. Nat Biotechnol 2011 29:762-766.

27. Fan JB, Oliphant A, Shen R, Kermani BG, Garcia F, Gunderson KL, Hansen M, Steemers F, Butler SL, Deloukas P, Galver L, Hunt S, McBride C, Bibikova M, Rubano T, Chen J, Wickham E, Doucet D, Chang W, Campbell D, Zhang B, Kruglyak S, Bentley D, Haas J, Rigault P, Zhou L, Stuelpnagel J, Chee MS: Highly parallel SNP genotyping. Cold Spring Harb Symp Quant Biol 2003, 68:69-78.

28. Fan JB, Chee MS, Gunderson KL: Highly parallel genomic assays. Nat Rev Genet 2006, 7:632-644.

29. Fan JB, Gunderson KL, Bibikova M, Yeakley JM, Chen J, Wickham Garcia E, Lebruska LL, Laurent M, Shen R, Barker D: Illumina universal bead arrays. Methods Enzymol 2006b, 410:57-73.

30. Akhunov E, Nicolet C, Dvorak J: Single nucleotide polymorphism genotyping in polyploid wheat with the Illumina GoldenGate assay. Theor Appl Genet 2009, 119:507-517.

31. Luo MC, Xu K, Ma Y, Deal KR, Nicolet CM, Dvorak J: A high-throughput strategy for screening of bacterial artificial chromosome libraries and anchoring of clones on a genetic map constructed with single nucleotide polymorphisms. BMC Genomics 2009, 10:28.

32. Bach K: Duplizierte Gene in Brassica napus - genetische Vielfalt in Kandidatengenen für Ölgehalt. PhD thesis. University of Potsdam: Faculty of Mathematics and Natural Sciences; 2007.

33. Klein PE, Klein RR, Cartinhour SW, Ulanch PE, Dong J, Obert JA, Morishige DT, Schlueter SD, Childs KL, Ale M, Mullet JE: A high-throughput AFLPbased method for constructing integrated genetic and physical maps: progress toward a sorghum genome map. Genome Res 2000, 10:789-807.

34. Cheng F, Liu S, Wu J, Fang L, Sun S, Liu B, Li P, Hua W, Wang X: BRAD, the genetics and genomics database for Brassica plants. BMC Plant Biol 2011 11:136. http://brassicadb.org/brad/.
35. Sambrook J, Fitsch EF, Maniatis T: Molecular Cloning: A Laboratory Manual. Cold Spring Harbor: Cold Spring Harbor Press; 1989.

36. Arumuganathan K, Earle ED: Nuclear DNA content of some important plant species. Plant Mol Biol Rep 1991, 9:208-218.

37. Ostergaard L, King GJ: Standardized gene nomenclature for the Brassica genus. Plant Methods 2008, 4:10.

38. Brudno M, Steinkamp R, Morgenstern B: The CHAOS/DIALIGN WWW server for multiple alignment of genomic sequences. Nucleic Acids Res 2004, 32:W41-44. http://dialign.gobics.de/chaos-dialign-submission.

39. Rozen S, Skaletsky H: Primer3 on the WWW for general users and for biologist programmers. Methods Mol Biol 2000, 132:365-386.

40. Hall T: BioEdit. http://www.mbio.ncsu.edu/BioEdit/bioedit.html.

doi:10.1186/1471-2164-14-603

Cite this article as: Cao and Schmidt: Screening of a Brassica napus bacterial artificial chromosome library using highly parallel single nucleotide polymorphism assays. BMC Genomics 2013 14:603.

\section{Submit your next manuscript to BioMed Central and take full advantage of:}

- Convenient online submission

- Thorough peer review

- No space constraints or color figure charges

- Immediate publication on acceptance

- Inclusion in PubMed, CAS, Scopus and Google Scholar

- Research which is freely available for redistribution

Submit your manuscript at www.biomedcentral.com/submit
C) Biomed Central 